\title{
A tool for technical and economic evaluation of dieback in wine-growing holdings
}

\author{
H. Mahé1 ${ }^{1}$ C. Riou $^{2}$, P. Longepierre ${ }^{3}$, M.-H. Imberti ${ }^{1}$, L. Mahé ${ }^{4}$ et C. Prêtet-Lataste ${ }^{1}$ \\ ${ }^{1}$ IFV CNIV 12 rue Sainte Anne 75001 Paris, France \\ 2 IFV Domaine de l'Espiguette 30200 Le Grau du Roi, France \\ ${ }^{3}$ BIVB 12 boulevard Bretonnière 21200 Beaune, France \\ ${ }^{4}$ INSA 20 Avenue des Buttes de Coesmes, 35700 Rennes, France
}

\begin{abstract}
Dieback in the vineyards is causing a long-term decline in the productivity of the vine stock and/or its sudden or gradual premature death, related to a number of different factors. It is a problem in all wine regions around the world. Its economic importance was estimated to represent $€ 2$ billion turnover in 2020 if the trend in yield loss continues at the same pace. How do we diagnose dieback in the wine-growing holdings to provide a better understanding of the risks and to respond? The aim of the work is to identify the factors to be taken into consideration in the development of a dieback self-diagnosis tool for wine growers. The first study takes into account all aspects of the wine-growing system in very mixed French conditions of production. The survey was fielded in 124 wine-growing and contains over a hundred questions. The analysis and classification of the holdings are based on yield and longevity which are constructed from socio-technical and economic data, complementing the agronomic aspects. These parameters have been combined to create a classification of wine-growing holdings affected by dieback. The finished tool is based on the answers to 30 questions identified by the survey. We can calculate a score that places the winery in relation to other wineries in the same situation.
\end{abstract}

\section{Introduction}

Les dépérissements du vignoble se traduisent par une baisse pluriannuelle subie de la productivité du cep et/ou sa mort prématurée brutale ou progressive, liée à une multiplicité de facteurs biologiques, environnementaux et viticoles pouvant affaiblir le cep sur la durée et/ou déclencher un processus qui s'avère irréversible en l'absence de moyens de lutte [1]. Cette problématique concerne l'ensemble des vignobles européens et mondiaux.

En 2016, la filière viti-vinicole française a lancé un plan d'action national pour lutter contre le dépérissement $\mathrm{du}$ vignoble [2]. Ce plan d'envergure pour la filière a permis de lancer des travaux autour de quatre ambitions complémentaires et interdépendantes que sont la formation et l'accompagnement des viticulteurs, l'amélioration de l'approvisionnement en matériel végétal, la mise en place d'un observatoire du vignoble et lancement d'un plan de recherche [3].

Depuis 2017, une cinquantaine de formations permettent de former chaque année plus de 600 personnes à la gestion des dépérissements ; 350 viticulteurs se sont regroupés en réseaux pour travailler ensemble sur les maladies du bois, les viroses et le matériel végétal et 21 programmes de recherche sont financés permettant à 45 équipes de recherche d'améliorer les connaissances sur les dépérissements du vignoble.

On estime en France que les dépérissements sont responsables d'une baisse de rendement des AOP (Appellations d'Origine Protégée) de $4.6 \mathrm{hL} / \mathrm{ha}$ en 2014 , soit un manque à produire de 3.4 millions d'hectolitres pour l'ensemble du vignoble français [4]. La perte économique est estimée en France à 2 milliards d'euros de chiffre d'affaire en 2020 si les tendances de baisse de rendement se confirment. L'étude du bilan critique des connaissances [1] a permis d'élaborer les pistes et voies d'action préalable au lancement du Plan dépérissement [2]. Elle avait mis en évidence le manque de connaissances sur les liens entre les dépérissements et les facteurs socioéconomiques des exploitation (structure des exploitations touchées, choix des investissements et des itinéraires techniques, changement des pratiques, évolution de la marge et du stock, etc.).

Afin d'évaluer plus finement l'impact économique des dépérissements à l'échelle des exploitations, une enquête a été conduite en 2017 dans des exploitations-type. Cette nouvelle étude a conduit à la mise au point d'un outil de diagnostic des dépérissements puis au développement d'un outil d'autoévaluation. Désormais, chaque exploitation peut évaluer son risque de dépérissement et se comparer ainsi avec les exploitations de sa région. Les différentes étapes de ces travaux sont présentées ci-après.

\section{Enquête socio-économique et évaluation des dépérissements}

\subsection{Méthodologie de l'étude}

Tout d'abord, afin de mieux comprendre l'impact des dépérissements à l'échelle des exploitations, une enquête d'envergure a été menée via des questionnaires en ligne. 400 exploitations ont répondu à cette première vague 




Figure 1. Définition du dépérissement.

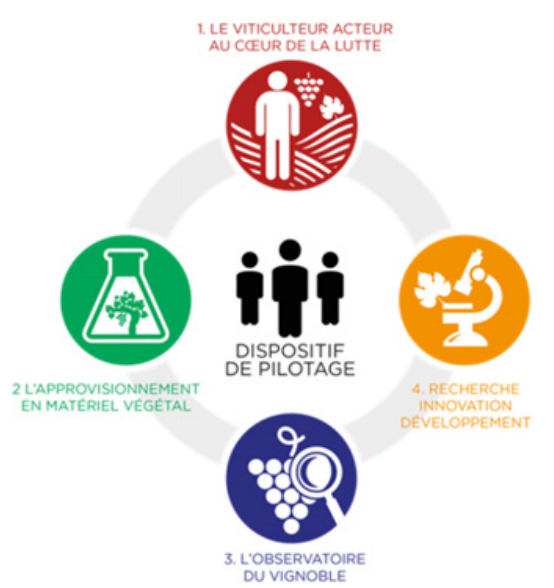

Figure 2. Les quatre ambitions du Plan Dépérissement.

d'enquête. Les exploitations étaient réparties dans cinq bassins de production : Bordeaux, Bourgogne, Beaujolais, Val de Loire et Vallée du Rhône. L'étude a pris en compte tous les aspects du système d'exploitation viticole dans des conditions de production contrastées. Cette enquête qui comporte plus d'une centaine de questions a permis de dresser un tableau complet de 124 exploitations viticoles qui ont répondu intégralement au questionnaire [5]. Les données collectées sont les suivantes :

- Portrait du chef d'exploitation et de sa formation,

- Structure de l'exploitation : surface de vigne, encépagement, appellations, type de production, niveau de valorisation...

- Éléments comptables : chiffres d'affaires et son évolution, postes d'investissement, niveau d'endettement, répartition des charges

- Description du matériel végétal

- Modes de gestion du vignoble

- Description des itinéraires techniques à l'échelle de la parcelle (densité, taux de manquants, taux de mortalité)

- Main d'œuvre

- Sous-traitance.

Dans ce questionnaire très complet les viticulteurs décrivaient la situation de leur exploitation puis étaient interrogés sur une parcelle type représentant la situation moyenne de leur exploitation. On a ainsi considéré dans la suite de ce travail que les valeurs mesurées sur une parcelle représentative de l'exploitation représentent la situation de toute l'exploitation vis-à-vis des dépérissements. L'échelle de l'exploitation a donc été considérée au travers du prisme d'une parcelle type de l'exploitation.

Statistiquement, la base des 124 réponses est modeste mais elle s'avère suffisante pour tirer des enseignements clefs dans la compréhension des dépérissements en lien avec les choix faits à l'échelle de l'exploitation. Cette base

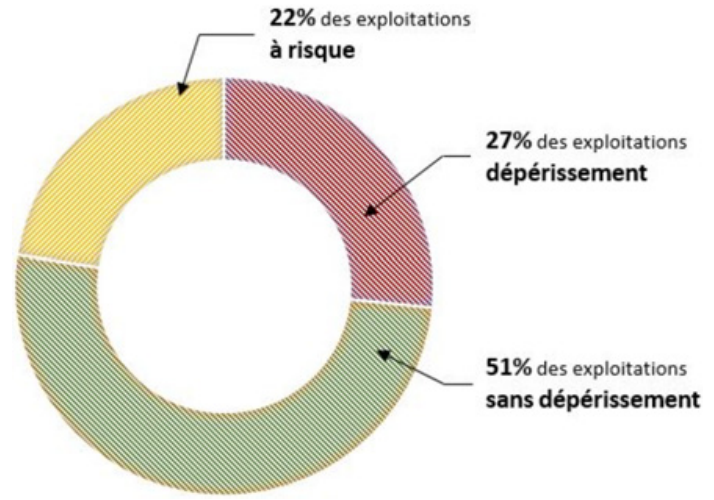

Figure 3. Répartition des exploitations en fonction du risque de dépérissement.

a également permis la mise au point d'une méthodologie d'estimation des dépérissements à l'échelle d'une parcelle.

L'analyse et la classification des exploitations sont basées sur les deux paramètres centraux dans le dépérissement que sont le rendement et la longévité. Ces deux notions de production sont construites à partir de données sociotechniques et économiques, complétant les aspects agronomiques à l'échelle du cep et de la parcelle. Elles traduisent des stratégies de production différentes.

\subsection{Répartition des exploitations enquêtées par rapport à leur risque de dépérissement}

Les dimensions rendement et longévité ont été combinées afin de constituer une classification d'exploitations selon leur niveau d'atteinte par les dépérissements. Elle distingue trois catégories :

- les exploitations peu ou pas sensibles au dépérissement,

- les exploitations atteintes par les dépérissements,

- les situations intermédiaires pour lesquels des dépérissements sont présents sans que la situation soit problématique.

Parmi les exploitations enquêtées, $51 \%$ sont identifiées comme peu ou pas sensibles. Elles atteignent leurs objectifs de rendement et de longévité de la parcelle. A l'inverse $49 \%$ présentent un risque ou une situation de dépérissement avérée. Ce sont ces situations, diverses, qu'il s'agit d'identifier et d'analyser.

\subsubsection{Perception des viticulteurs et situation par rapport aux dépérissent dépérissement}

$66 \%$ des viticulteurs enquêtés se disent touchés par le dépérissement même s'ils atteignent leurs objectifs de rendement. Ce chiffre témoigne de la préoccupation qu'engendre le dépérissement mais aussi de la nécessité de mieux appréhender cette notion.

A contrario, plusieurs exploitations semblent présenter un risque de dépérissement alors que les viticulteurs ne le perçoivent pas, confirmant l'intérêt de la mise en place d'un outil d'autodiagnostic à l'échelle de l'exploitation.

\subsection{Tris à plat et choix des variables représentatives}

Afin de sélectionner les variables les plus significatives dans l'estimation du rendement et de la longévité, des tris 


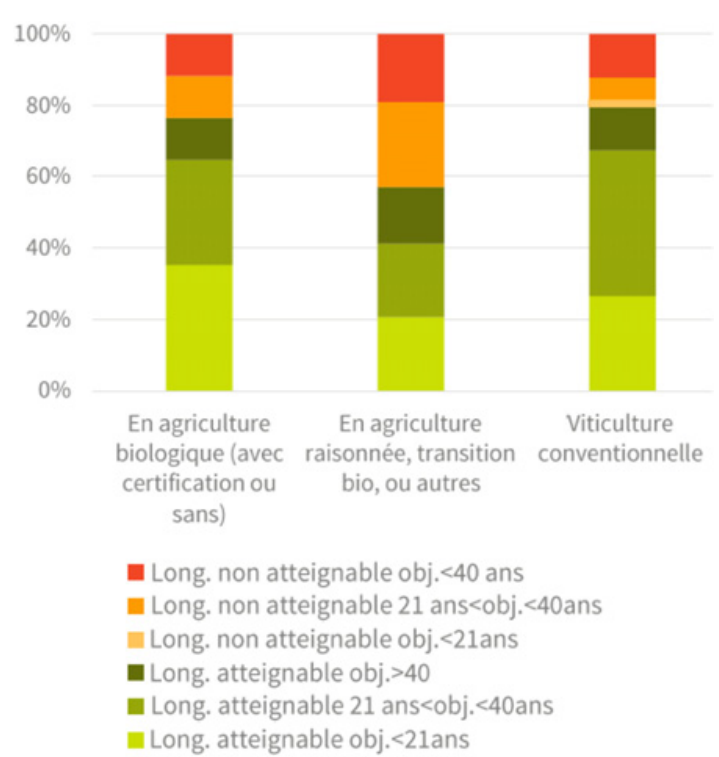

Figure 4. Objectifs de longévité en fonction de la conduite de la vigne.

à plat et des tests $\chi^{2}$ ont été réalisés. La significativité de toutes les variables a été testée. Seuls les résultats sur les variables significatives sont présentés ci-après :

- Système de culture

- Situation financière des exploitation

- Statut de l'exploitation

- Durée de la période de taille.

\subsubsection{Dépérissement et systèmes de culture}

Le niveau d'exposition au risque de dépérissement apparaît supérieur au sein des exploitations n'ayant pas encore fait un choix unique sur leur système de culture. Il s'agit d'exploitations se situant entre deux modalités de système de culture (transition vers l'agriculture biologique ou viticulture raisonnée) ou de mode de faire-valoir principal (modèle locataire versus modèle patrimonial).

La Fig. 4 illustre l'atteinte des objectifs de longévité par les exploitations en fonction de leur système de culture (biologique, raisonné ou conventionnel).

Cette surexposition au dépérissement pour les exploitations en transition ou en viticulture raisonnée peut s'expliquer par la complexité accrue à piloter les parcelles, l'absence d'un référentiel unique et la difficulté à trouver un équilibre entre rendement et longévité.

\subsubsection{Dépérissement et statut de production}

Le statut coopérateur semble diminuer la sensibilité des exploitations au dépérissement. D'avantage positionnés sur des objectifs de rendements moyens à hauts, les coopérateurs connaissent cependant moins de situations de non atteinte du rendement. Cependant, bien qu'ils concentrent leur activité sur la gestion du vignoble et bénéficient fréquemment des conseils agronomiques d'un technicien de la coopérative, leur situation est comparable à celle des autres exploitations en termes de risque sur la longévité des parcelles.
Tableau 1. Attribution des scores sur la dimension rendement.

\begin{tabular}{|l|c|c|c|}
\hline Objectif (années) & $\begin{array}{c}\leq \mathrm{à} 40 \\
\mathrm{hL} / \mathrm{ha}\end{array}$ & $\begin{array}{c}\text { entre } 41 \text { et } 60 \\
\mathrm{hL} / \mathrm{ha}\end{array}$ & $\begin{array}{c}>60 \\
\mathrm{hL} / \mathrm{ha}\end{array}$ \\
\hline Valeur attribuée & 0 & 1 & 2 \\
\hline $\begin{array}{l}\text { Malus si l'objectif } \\
\text { n'est pas atteignable }\end{array}$ & $0+0=0$ & $0+1=1$ & $0+2=2$ \\
\hline $\begin{array}{l}\text { Bonus si l'objectif est } \\
\text { atteignable }\end{array}$ & $3+0=3$ & $3+1=4$ & $3+2=5$ \\
\hline
\end{tabular}

\subsubsection{Dépérissement et situation financière des exploitations}

Les liens entre le dépérissement et la situation financière des exploitations viticoles sont également perceptibles. Ainsi, lorsque le taux de marge brute baisse depuis plusieurs années ou que les exploitations présentent des niveaux d'endettement soit trop forts soit trop faibles, elles sont aussi plus souvent exposées aux risques de dépérissement de leurs parcelles. Ces niveaux opposés peuvent traduire une cause du dépérissement (manque d'investissement), mais aussi une conséquence (hausse des charges et baisse des recettes). Les exploitations visant un faible niveau de valorisation depuis plusieurs années peuvent être particulièrement sensibles : elles ont une faible marge et sont par conséquent très dépendantes du niveau de rendement qu'elles peuvent atteindre pour générer du résultat.

\subsubsection{Dépérissement et période de taille}

L'allongement de la durée de la période de taille est un indice révélateur des exploitations à risque élevé de dépérissement. Cette durée est liée à la disponibilité de la main d'œuvre et aux incidents climatiques. Lorsqu'ils sont touchés par les dépérissements, les viticulteurs enquêtés s'orientent vers des opérations viticoles spécifiques pour soutenir la longévité de la vigne. Le nombre de ces opérations est un bon indicateur de l'exposition des exploitations au dépérissement.

\subsection{Rendement et longévité au cœur de la problématique dépérissement}

Comme déjà explicité, le rendement et la longévité sont au cœur de la problématique de dépérissement [1]. Afin d'estimer le risque de dépérissement, on détermine une classification pour le rendement et une autre pour la longévité. Ces classifications sont enrichies par un score en fonction de l'atteinte ou non d'un valeur seuil $(80 \%$ de l'objectif pour le rendement et $40 \%$ de l'objectif pour la longévité).

\subsubsection{Estimation de la dimension rendement}

L'estimation de la dimension rendement est basée sur un raisonnement simple. On attribue une valeur en fonction de l'objectif de rendement que se fixe le viticulteur (cf Tableau 1):

- Moins de $40 \mathrm{hL} / \mathrm{ha}$

- Entre 41 et $60 \mathrm{hL} / \mathrm{ha}$

- Plus de $60 \mathrm{hL} / \mathrm{ha}$. 
Tableau 2. Calcul du score de longévité.

\begin{tabular}{|c|c|}
\hline Objectif & Valeur attribuée \\
\hline$<21$ ans & 0 \\
\hline entre 21 et 40 ans & 1 \\
\hline entre 41 et 60 ans & 2 \\
\hline$>60$ ans & 3 \\
\hline
\end{tabular}

Si cet objectif est au moins atteint à $80 \%$, un bonus de 3 points est attribué (si ce n'est pas le cas, aucun bonus n'est ajouté). On obtient alors une dimension rendement dont la valeur est comprise entre 0 et 5 .

\subsubsection{Calcul de la classe de longévité :}

La classe de longévité est déterminée par le raisonnement suivant. Tout d'abord, on évalue quel est l'objectif de longévité de la parcelle. Ce calcul est simple et basé sur l'équation :

\section{Objectif de longévité}

= année courante - année de plantation

+ nombre d'annes d'exploitation supplémentaire

Ainsi, l'objectif de longévité en 2019 pour une parcelle plantée en 2002 avec un viticulteur qui souhaite encore exploiter sa parcelle pendant 40 ans est estimé à 57 ans $(2019-2002+40=57$ ans $)$.

Une première valeur est attribuée en fonction de l'objectif de longévité fixé. Ces valeurs sont attribuées selon la distribution suivante :

Dans l'exemple, la valeur attribuée sera de 2.

On détermine ensuite le nombre d'années restant théoriquement à la parcelle, c'est-à-dire le nombre d'années de longévité avant que la parcelle ne soit arrachée $\mathrm{du}$ fait d'un nombre de manquants ou d'improductifs trop important. Soit N ce nombre d'années de longévité restant. Il est posé que cette date est atteinte lorsque $40 \%$ des pieds initiaux de la parcelle sont manquants et en supposant qu'aucune action n'est entreprise pour les remplacer. La durée de vie théorique de la parcelle se calcule par l'équation suivante :

$$
\begin{aligned}
& \text { nombre de Pieds } *(1-\text { taux de mortalité })^{N+1}= \\
& \text { nombre Pieds } * 0,6 \\
& (1-\text { taux de mortalité })^{N+1}=0,6 \\
& (N+1) * \log (1-\text { taux de mortalité })=\log 0,6
\end{aligned}
$$

$$
\text { Soit } \mathrm{N}=\frac{\log (0,6)}{\log (1-\text { taux de mortalité })}-1
$$

Le taux de mortalité sur la parcelle est estimé par le calcul suivant :

Taux de mortalité $=$

nombre de pieds morts

surface parcelle $*$ densité Pieds $*((1-$ tauxManquants $) / 100)$

L'objectif est donc atteignable si $\mathrm{N}$ (le nombre d'années restant à la parcelle) est supérieur au nombre

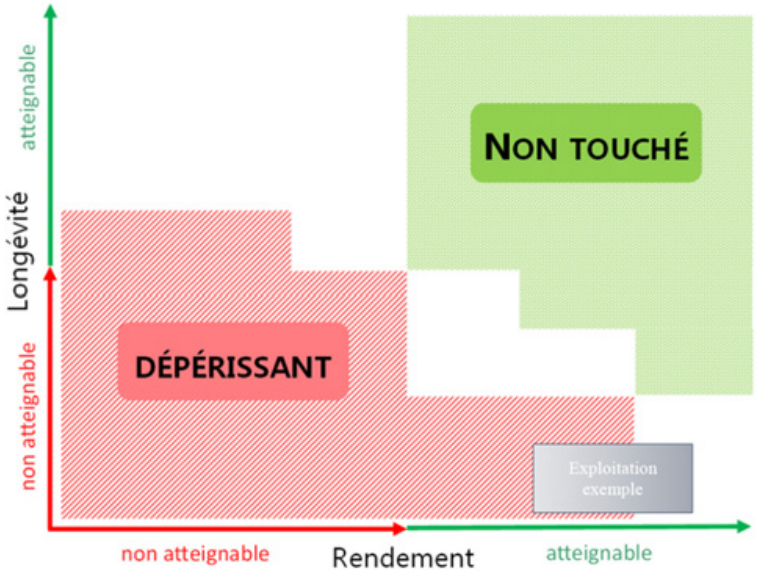

Figure 5. Matrice des typologies de dépérissement.

d'années d'exploitation supplémentaires espéré par le viticulteur avant arrachage.

Ainsi, dans une parcelle de 2 ha avec une densité de 6400 pieds à l'hectare et dont 600 pieds sont morts au cours de la dernière année, le taux de mortalité s'élève à $4.9 \%$. La longévité espérée de la parcelle est alors estimée à 9 ans avant d'atteindre $40 \%$ de ceps absents. L'objectif visé par l'exploitant, qui était de maintenir sa parcelle encore 40 ans, n'est pas atteignable.

$\mathrm{Au}$ score d'objectif de longévité précédemment attribué s'ajoute un bonus ou un malus en fonction de la possibilité d'atteindre l'objectif. Le score final qualifiant la longévité a donc une valeur comprise entre 0 et 7 en effectuant la somme de deux chiffres obtenus via le Tableau 3 ci-dessous :

Le bonus est attribué si l'objectif est supérieur à la durée de vie théorique. Dans l'exemple, l'objectif de longévité n'étant pas atteignable, le score longévité reste fixé à 2.

\subsection{Estimation du risque de dépérissement}

Les scores obtenus pour le rendement et la longévité sont croisés afin de déterminer un positionnement d'exploitations plus ou moins atteintes par les dépérissements (cf. Fig. 5).

Cette combinaison est construite selon deux dimensions et distingue trois classes en fonction de la matrice des typologies:

- les exploitations moins sensibles au dépérissement (ie non touchées par le dépérissement),

- les exploitations atteintes par les dépérissements,

- les situations intermédiaires (exploitations qui ne sont ni classées comme indemnes de dépérissement ni comme dépérissantes).

Dans l'exemple, l'exploitation analysée obtient un score de 4 sur le rendement (objectif de rendement atteint à $90 \%$ ) mais de 2 sur la longévité, ce qui la place dans les exploitations à risque de dépérissement. Cette exploitation est à risque de dépérissement, puisqu'elle n'atteint pas son objectif de longévité. 
Tableau 3. Attribution des scores sur la dimension longévité.

\begin{tabular}{|l|c|c|c|c|}
\hline Objectif (années) & Moins de 21 ans & Entre 21 et 40 ans & Entre 41 et 60 ans & Plus de 61 ans \\
\hline Valeur attribuée & 0 & 1 & 2 & 3 \\
\hline Malus si l'objectif n'est pas atteignable & $0+0=0$ & $0+1=1$ & $0+2=2$ & $0+3=3$ \\
\hline Bonus si l'objectif est atteignable & $4+0=4$ & $4+1=5$ & $4+2=6$ & $4+3=7$ \\
\hline
\end{tabular}

\section{Méthode de calcul du score dépérissement}

Une fois les dimensions rendement et longévité estimées pour toutes les exploitations enquêtées, le score dépérissement a été établi par des calculs d'odds ratio qui permettent de mesurer l'effet d'un facteur sur deux populations distinctes $[6,7]$. L'odds ratio, également appelé rapport des chances, rapport des cotes ou risque relatif approché est une mesure statistique permettant de mesurer le degré de dépendance entre des variables qualitatives.

\subsection{Calcul des odds-ratio sur les 8 variables significatives}

Les tris à plat et les tests de tests de $\chi^{2}$ nous ont permis de sélectionner les 8 variables pertinentes car statistiquement significatives. Les calculs d'odds ratio sont alors effectués pour chacune des 8 variables retenues pour le calcul du score :

- Part de la récolte livrée en coopérative

- Évolution de la marge

- Dépenses en produits phytosanitaires

- Taux d'endettement

- Nombre d'opérations culturales adoptées pour soutenir la longévité

- Niveau d'exigence environnementale du mode de conduite de la vigne

- Durée de la phase de taille

- Niveau de diplôme de l'exploitant.

Ces valeurs d'odds ratio sont sommées pour obtenir un score, correspondant à une exploitation enquêtée. Le calcul est le suivant :

- On pose p la proportion d'individus de type dépérissant touchée par un événement $\mathrm{A}$, par exemple le fait que l'exploitant apporte plus de la moitié de sa récolte en cave coopérative

- On pose q la proportion d'individus de type nondépérissant touchée par ce même événement A

L'odds ratio est alors :

$$
\text { Odds Ratio }=\frac{\frac{p}{1-p}}{\frac{q}{1-q}}
$$

Ainsi plus cette valeur est élevée, plus l'événement A est fréquent dans la population des exploitations en situation de dépérissement.

La distribution des scores totaux calculés parmi les 124 exploitations est présentée en Fig. 9. Plus le score est élevé, plus le risque de dépérissement est important sur la parcelle utilisée comme référence.

Une fois la somme d'odds ratio calculée, il est possible de représenter les scores de chaque exploitation en

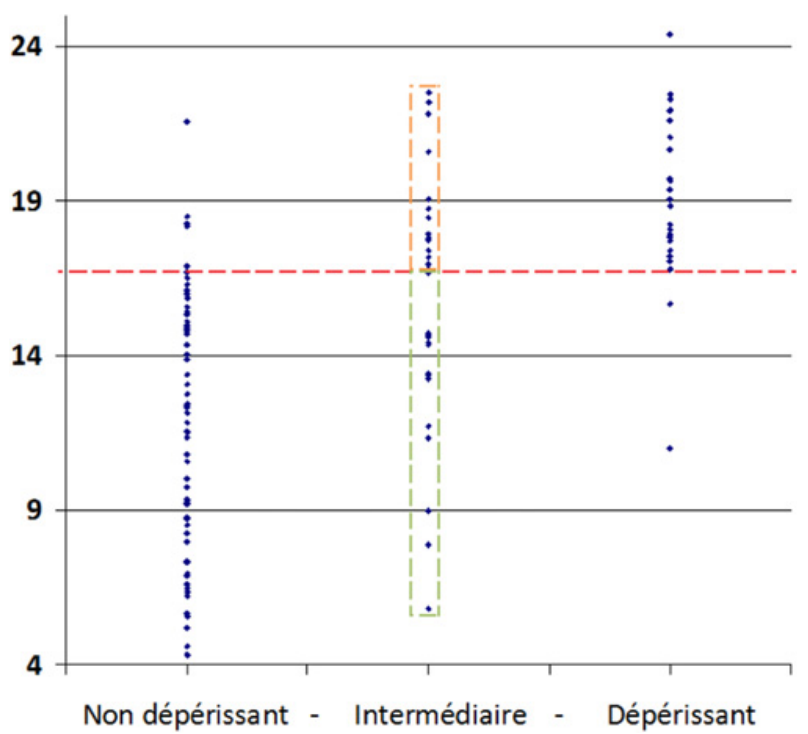

Figure 6. Scores et classes de dépérissement : un seuil séparant au mieux les populations dépérissantes et non touchées peut être tracé.

fonction des trois classes de dépérissement. Le graphique ainsi obtenu et représenté en Fig. 6 vise al obtenir une meilleure précision quant aux exploitations déterminées de classe intermédiaire.

\subsection{Une amélioration nécessaire des odds-ratio}

L'enquête et le traitement des données collectées ont permis de mettre au point la méthode de calcul du score de dépérissement constituant une première base de référence. Cependant, cette base mérite d'être consolidée sur un plus grand nombre d'exploitations, ce qui permettrait d'avoir une classification plus franche entre les exploitations dépérissantes et les exploitations non dépérissantes.

Cette étude a donc démontré la pertinence d'une approche à $360^{\circ}$ de l'exploitation, intégrant des éléments sociotechniques et socioéconomiques. Elle conclut à la faisabilité d'un outil d'autodiagnostic des dépérissements à l'échelle de l'exploitation. Elle mérite d'être renforcée par ce type d'outil qui permettra d'améliorer la robustesse des résultats.

\section{Mise au point de l'outil d'auto-évaluation et premiers résultats}

Un outil d'auto-évaluation a donc été développé pour permettre aux viticulteurs d'estimer leur risque de dépérissement en fonction de paramètres facilement mesurables [7]. 


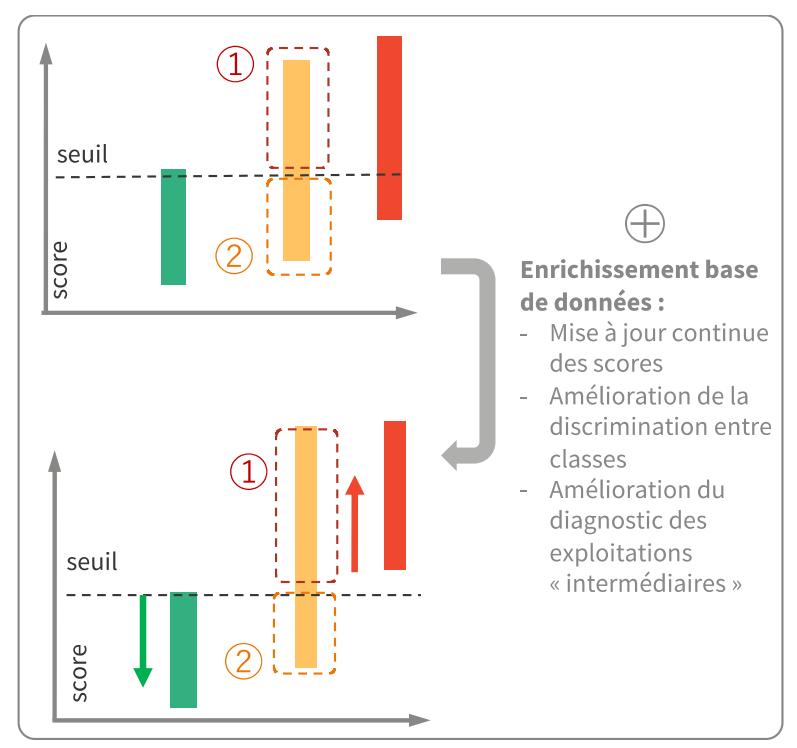

Figure 7. Amélioration attendue des odds-ratio.

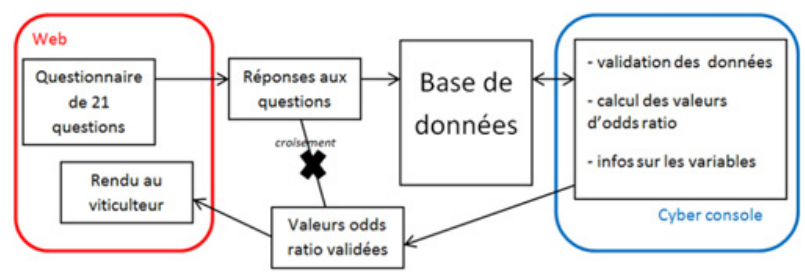

Figure 8. Schéma de la structure de l'outil d'auto-évaluation.

\subsection{Fonctionnement de l'outil d'auto-évaluation}

Le questionnaire initial a été simplifié et restreint aux 30 questions les plus discrimantes en lien avec les 8 variables déterminantes afin d'établir un diagnostic dépérissement.

L'outil d'auto-évaluation vient compléter les résultats précédemment acquis en alimentant une base de données commune. L'organisation et le fonctionnement de l'outil sont précisés sur la figure suivante.

L'outil d'auto-évaluation permet de distinguer les exploitations viticoles selon trois profils :

- exploitations pas ou peu exposées au dépérissement ;

- exploitations à risque élevé, partiellement ou non identifié par le viticulteur et nécessitant une analyse plus approfondie ;

- situations caractéristiques des exploitations touchées par le dépérissement.

Les calculs de classe longévité et rendement et d'odds ratio s'effectuent en temps réel et un rapport d'évaluation est généré et mis à disposition du viticulteur.

L'outil a été testé sur des exploitations bourguignonnes afin de vérifier la pertinence du classement par type d'exploitation et sa robustesse.

\subsection{Premiers résultats obtenus}

L'outil a été lancé le 15 février 2019. Il est mis en ligne directement sur le site Internet du Plan dépérissement [9].

Trois mois après son lancement, 20 questionnaires complets ont été renseignés et ont permis de réaliser des calculs d'odds-ratio.

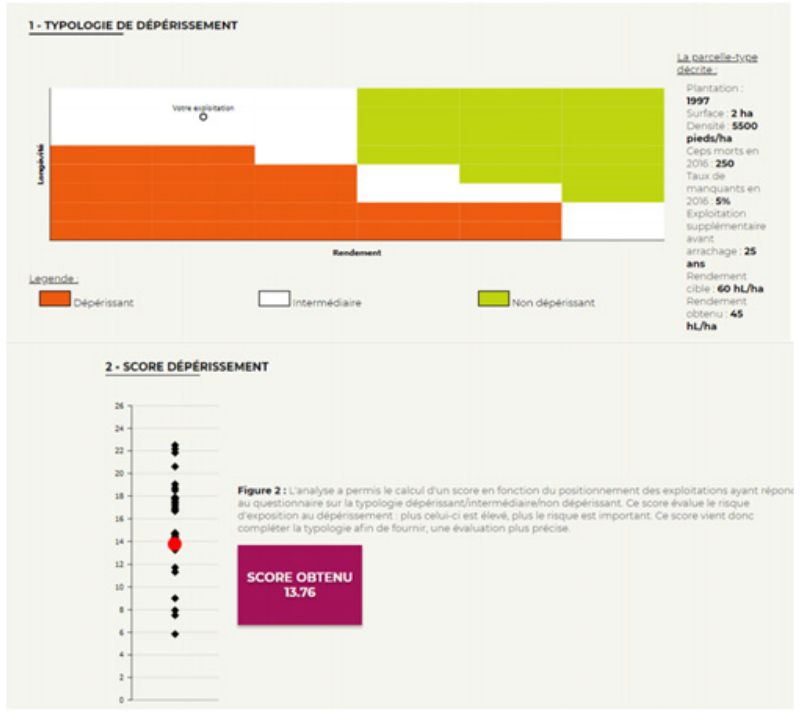

Figure 9. Rapport d'évaluation généré automatiquement par l'outil d'auto-évaluation.

\subsubsection{Typologie des exploitations ayant réalisé un auto-diagnostic}

Premier résultat positif de l'outil, l'auto-diagnostic a été testé dans la plupart des régions viticoles françaises. Des réponses provenant d'Alsace, du Jura, de Charente ou du Sud-Ouest viennent compléter les réponses obtenues précédemment.

On constate en moyenne que ces 20 exploitations présentent une densité de plantation moyenne de 6000 pieds/ha, un taux de manquant de $12 \%$ et un âge moyen de 29 ans. Les exploitations interrogées en 2017 2018 avaient plutôt une densité moyenne déclarée de 6400 pieds/ha, un taux de manquants de $6 \%$ et un âge moyen de 27 ans. Les deux échantillons sont donc similaires, les valeurs obtenues par l'outil d'auto-évaluation sont en cohérence par rapport aux données précédemment acquises. De même, la réduction du nombre de questions semble ne pas avoir d'impact dans les résultats obtenus.

\subsubsection{Comparaison des premiers scores obtenus}

La figure suivante compare les scores de dépérissement obtenus par les entreprises enquêtées dans l'étude initiale avec ceux obtenus par l'outil d'auto-diagnostic en ligne.

La représentation en «boîte à moustaches » (Fig. 10) permet de montrer la répartition des valeurs autour de la valeur médiane. Les bords du rectangle délimitent les valeurs comprises entre le premier et le troisième quartile. Ce type de représentation est particulièrement utile pour comparer un même caractère dans deux populations différentes [10].

Les viticulteurs qui réalisent le test d'auto-évaluation semblent donc directement concernés par les dépérissements puisque les score sont plus concentrés. La valeur médiane dans le nouvel échantillon est supérieure à la valeur médiane obtenue dans la première enquête.

\section{Conclusion}

Ce travail d'enquête a permis la conception d'un outil d'auto-évaluation qui fonctionne désormais. L'outil est 


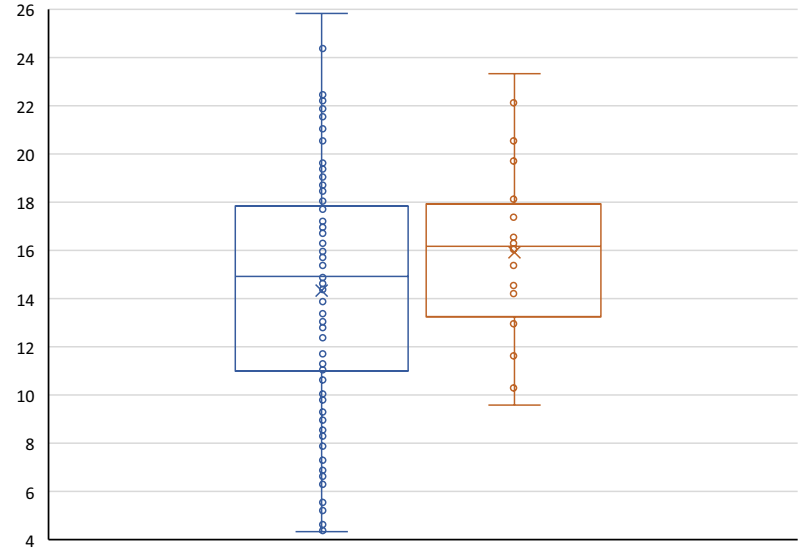

$\square$ Score de dépérissement pour les 124 premières exploitations

$\square$ Score de dépérissement pour les exploitations ayant répondu à I'OAE

Figure 10. Comparaison de la répartition des scores «dépérissement» parmi les questionnaires de l'enquête initiale et ceux de l'outil d'auto-évaluation.

accessible via le site Internet du Plan Dépérissement. Il a été conçu pour accompagner les viticulteurs dans leur évaluation des dépérissements sur leur exploitation. Très simple d'utilisation, il permet en moins de 10 minutes d'obtenir une réponse précise sur la situation de l'exploitation par rapport aux dépérissements. L'outil est également disponible via les sites techniques des interprofessions de vin. Il est également mis à disposition des techniciens et conseillers (Chambres d'agriculture, instituts techniques, cabinets de conseil etc.). Il peut leur permettre de détecter les exploitations nécessitant un accompagnement spécifique pour lutter efficacement contre les dépérissements. L'outil est également inclus dans des formations numériques (webformation du plan dépérissement par exemple) ou des formations en présentielle. Dans ce cas, il est utilisé comme outil de sensibilisation à la problématique des dépérissements.

L'outil d'auto-évaluation permettra à terme de comparer sa situation personnelle au regard d'exploitations du même type dans le même bassin de production.

Outil dynamique, l'autodiagnostic viendra prochainement alimenter l'infrastructure de données géographiques développée dans le cadre de l'observatoire du vignoble. Il pourra à terme permettre d'évaluer des choix d'itinéraires techniques ou d'évolutions de l'exploitation, au regard du risque de dépérissement. Son utilisation fréquente et par de nombreux exploitants permettra de mieux accompagner la filière sur cette problématique du dépérissement. Il conduira également au développement d'outil d'aide à la décision plus spécifique sur les choix d'itinéraires culturaux par exemple.

\section{Références}

[1] C. Riou, D. Agostini, P. Aigrain, M. Barthe, ML. des Robert, JP. Gervais, E. Jobard, L. Lurton, D. Moncomble et C. Prêtet-Lataste, Web of Conferences 39th OIV Congress (2016)

[2] France AgriMer CNIV BIPE Rapport final de l'étude réalisée par le BIPE dans le cadre du Plan national de lutte contre les dépérissements du vignoble (2016) Présentation du Plan national de lutte contre les dépérissements du vignoble (2016)

[3] Plan National Dépérissement du Vignoble - site institutionnel www.plan-deperissement-vigne.fr

[4] France AgriMer CNIV BIPE Equipe projet constituée de Vianney Da Costa, Etienne Jobard, Nathalie Leridon, Giulia Manfroni, Jacques Marquay, Stéphane Radureau et Perrine Tonin, sous la direction de Marie-Laetitia des Robert, avec l'appui technique d'Albane Cosson. Etude BIPE FAM-CNIV, Dépérissement du vignoble (2015)

[5] France AgriMer CNIV BIPE Analyse socioéconomique des dépérissements 88 p (2018)

[6] P-M. Bernard et C. Lapointe Mesures statistiques en épidémiologie. Presses de l'Université du Québec, Sainte-Foy (1995)

[7] A. Jammal, G. Loslier, R. Allard, Dictionnaire d'épidémiologie, Edisem/Maloine, St-Hyacinthe/ Paris, p. 124-125 1988)

[8] L. Mahé, Analyse socio-économique des dépérissements du vignoble- Rapport de stage $23 p$ (2018)

[9] L. Mahé, H. Mahé, Outil d'auto-évaluation des dépérissements sur l'exploitation : https://app. plan-deperissement-vigne.fr/\#/infos

[10] M. Le Guen, «La boîte à moustaches pour sensibiliser à la statistique», Bulletin de méthodologie sociologique 73, 43 (2002) 